\title{
IRE1 Alpha/XBP1 Axis Sustains Primary Effusion Lymphoma Cell Survival by Promoting Cytokine Release and STAT3 Activation
}

\author{
Roberta Gonnella ${ }^{1,2}$, Maria Saveria Gilardini Montani ${ }^{1,2} \mathbb{D}$, Luisa Guttieri ${ }^{2,3} \mathbb{D}^{\mathbb{D}}$, Maria Anele Romeo ${ }^{1,2}$, \\ Roberta Santarelli ${ }^{1,2}$ and Mara Cirone ${ }^{1,2, *(D)}$ \\ 1 Department of Experimental Medicine, Sapienza University of Rome, 00161 Rome, Italy; \\ roberta.gonnella@uniroma1.it (R.G.); mariasaveria.gilardinimontani@uniroma1.it (M.S.G.M.); \\ mariaanele.romeo@uniroma1.it (M.A.R.); roberta.santarelli@uniroma1.it (R.S.) \\ 2 Laboratory Affiliated to Istituto Pasteur Italia-Fondazione Cenci Bolognetti, 00161 Rome, Italy; \\ luisa.guttieri@uniroma1.it \\ 3 Department of Clinical and Molecular Medicine, Sapienza University of Rome, 00161 Rome, Italy \\ * Correspondence: mara.cirone@uniroma1.it
}

Citation: Gonnella, R.; Gilardini Montani, M.S.; Guttieri, L.; Romeo, M.A.; Santarelli, R.; Cirone, M. IRE1 Alpha/XBP1 Axis Sustains Primary Effusion Lymphoma Cell Survival by Promoting Cytokine Release and STAT3 Activation. Biomedicines 2021, 9, 118. https://doi.org/10.3390/ biomedicines 9020118

Academic Editor: Kwang Ahn Received: 31 December 2020 Accepted: 25 January 2021 Published: 27 January 2021

Publisher's Note: MDPI stays neutral with regard to jurisdictional claims in published maps and institutional affiliations.

Copyright: (c) 2021 by the authors. Licensee MDPI, Basel, Switzerland. This article is an open access article distributed under the terms and conditions of the Creative Commons Attribution (CC BY) license (https:// creativecommons.org/licenses/by/ $4.0 /)$.

\begin{abstract}
Primary Effusion Lymphoma (PEL) is a highly aggressive B cell lymphoma associated with Kaposi's Sarcoma-associated Herpesvirus (KSHV). It is characterized by a high level of basal Endoplasmic Reticulum (ER) stress, Unfolded Protein Response (UPR) activation and constitutive phosphorylation of oncogenic pathways such as the Signal Transducer and activator of Transcription (STAT3). In this study, we found that the inositol requiring kinase (IRE) 1alpha/X-box binding protein (XBP1) axis of UPR plays a key role in the survival of PEL cells, while double stranded RNA-activated protein kinase-like ER kinase (PERK) and activating transcription factor (ATF) 6 slightly influence it, in correlation with the capacity of the IRE1alpha/XBP1 axis to induce the release of interleukin (IL)-6, IL-10 and Vascular-Endothelial Growth Factor (VEGF). Moreover, we found that IRE1alpha/XBP1 inhibition reduced STAT3 Tyr705 phosphorylation and induced a pro-survival autophagy in PEL cells. In conclusion, this study suggests that targeting the IRE1alpha/XBP1 axis represents a promising strategy against PEL cells and that the cytotoxic effect of this treatment may be potentiated by autophagy inhibition.
\end{abstract}

Keywords: ER stress; UPR; IRE1 alpha; XBP1; STAT3; cytokines

\section{Introduction}

Primary effusion lymphoma (PEL) is a highly aggressive B cell lymphoma whose etiology is strongly linked to Kaposi's Sarcoma-associated herpesvirus (KSHV) infection that is present in almost $100 \%$ of cases of PEL, in the majority of cases in association with Epstein-Barr virus (EBV). KSHV is a gammaherpesvirus that carries genes encoding for a variety of proteins involved in tumorigenesis such as viral Fas-associated with death domain (FADD)-like interleukin-1 $\beta$-converting enzyme (FLICE)/caspase 8-inhibitory protein (vFLIP) that is able to subvert the response to apoptotic stimuli and autophagy [1,2]. PEL presents in most cases as malignant lymphomatous effusions, mainly localized in body cavities, such as the pleural, pericardium and peritoneal cavities. PEL cells originate from postgerminal center late-differentiating B-cells [3]. The success of conventional therapies such as cyclophosphamide, doxorubicin, vincristine and prednisone (CHOP) against this lymphoma is very poor, which implies that the search for new and more effective treatments is urgent.

Autophagy, a catabolic process required for cellular homeostasis and response to stress, is important for cancer cell survival, especially in stressful conditions such as nutrient shortage or anticancer treatments. Autophagy inhibition in PEL cells in the course of several chemotherapies has been shown by our and others' laboratories to be a 
promising strategy to improve the outcome of such treatments [4,5]. Moreover, similarly to multiple myeloma (MM), PEL cells are highly secretory B cells and, therefore, are characterized by basal Endoplasmic Reticulum (ER) stress and Unfolded Protein Response (UPR) activation [6], which can be also increased by treatments, particularly the proteasome inhibitor Bortezomib [7]. Although a too high stress and too prolonged UPR activation may induce cell death, UPR is mainly a response that can help cells to survive by several means, i.e., by increasing chaperone expression, by blocking protein translation and degradation by proteasome and autophagy [8]. All these processes may be triggered by the single or overlapping functions of the three main UPR sensors, namely, inositol requiring kinase (IRE) 1 alpha, double stranded RNA-activated protein kinase-like ER kinase (PERK) and the activating transcription factor (ATF) 6, that indeed orchestrate an integrate response to stress. Regarding Ire1 alpha, this sensor may have kinase and endoribonuclease activities, generating in the latter case the spliced form of XBP1 (XBP1s), a molecule strongly involved in the survival of cancers such as MM [9]. Of note, UPR signaling affects multiple other processes, playing a key role in cellular and organism pathophysiology. UPR regulates, for example, the activation of molecular pathways such as NF-kB and STAT3 [10] that, among other functions, promote the release of cytokines and affect cancer cell survival as well as immune response [11]. Moreover, the antioxidant response, essential for maintaining the proper ROS balance required for the activation of pro-survival intracellular signaling, is also strongly influenced by UPR activation, particularly by PERK that may activate nuclear factor erythroid 2-related factor (NRF2) [12]. Given the central role of UPR in regulating multiple processes that ultimately dictate the cell fate, in this study, we investigated the possibility to reduce survival of PEL cells, characterized by a basal ER stress/UPR activation by inhibiting the tree UPR sensors PERK, IRE1alpha or ATF6. We also explored the impact of UPR sensor inhibition on autophagy, knowing that all these sensors have been shown to be involved in the activation of this catabolic process [13].

\section{Material and Methods}

\subsection{Cell Culture and Reagents}

BC3 (ATCC, CRL-2277) and BCBL1 (kindly provided by Prof. P. Monini, National AIDS Center, Istituto Superiore di Sanità, Rome, Italy) are human B-cell lines derived from Primary effusion Lymphoma (PEL). Cells were cultured in RPMI 1640 (SigmaAldrich, St. Louis, MO, USA, R0883) with $10 \%$ fetal calf serum (Euroclone, Milano, Italy, ECLS0180L), L-glutamine $(2 \mathrm{mM})$ streptomycin $(100 \mu \mathrm{g} / \mathrm{mL})$ and penicillin $(100 \mathrm{U} / \mathrm{mL})$ (Gibco, Gaithersburg, MD, USA, 10378-016) in $5 \% \mathrm{CO}_{2}$ at $37^{\circ} \mathrm{C}$. Cells were treated with the following drugs: $4 \mu 8 \mathrm{C}$ (IRE1 RNAse inhibitor) provided by Sigma (Sigma-Aldrich, MO, USA, cat n. SML0949), Ceapin-A7 (ATF6a signaling blocker) provided by Sigma-Aldrich (cat n. SML2330), GSK2606414 (PERK inhibitor) provided by Selleckem, USA (cat. n. S7307). Chloroquine (CQ) (inhibitor of autophagic protein degradation) (Sigma-Aldrich, MO, USA, cat. n. C6628). Chemicals were added to cell cultures at the final concentrations of 10 and $20 \mu \mathrm{M}(4 \mu 8 \mathrm{C}), 6$ and $12 \mu \mathrm{M}$ (Ceapin) and 10 and $20 \mathrm{ng} / \mathrm{mL}$ (GSK2606414) for $24 \mathrm{~h}$; Cloroquine was used at final concentration of $10 \mu \mathrm{M}$ for $18 \mathrm{~h}$. After treatments, cells were collected, counted by trypan blue exclusion assay using a hemocytometer and used for further analysis. Each experiment was performed in triplicate and repeated at least three times.

\subsection{Cell Viability}

Cell viability was evaluated by a trypan blue (Sigma-Aldrich, MO, USA) exclusion assay after $24 \mathrm{~h}$ of culture. Cells were counted by light microscopy using a Neubauer emocytometer. The experiments were performed in triplicate and repeated at least three times.

\subsection{Western Blot Analysis}

After treatments, cells were harvested, centrifuged and finally lysed in modified RIPA buffer (150 mM NaCl, 1\% NP40, $50 \mathrm{mM}$ Tris-HCl pH 8, 0.5\% deoxycholic acid, $0.1 \%$ 
SDS, $1 \%$ Triton $\mathrm{X}-100$ protease and phosphatase inhibitors) on ice for $30 \mathrm{~min}$ [14]. Protein concentration was determined by using a BCA protein assay kit (Sigma-Aldrich, MO, USA, $71285-\mathrm{M})$, and the same amount of each lysate $(8-10 \mu \mathrm{g})$ was loaded on $4-12 \%$ NuPage Bis Tris gels (Thermo Fisher, NP0323). Following electrophoresis, samples were blotted on Nitrocellulose membranes (Whatman, GE Healthcare, 10401196) that were blocked for 30 min at RT in PBS containing BSA 3\% and 0.2\% Tween 20, then probed with suitable primary antibody overnight at $4{ }^{\circ} \mathrm{C}$. After three washes with PBS $0.2 \%$ Tween, membranes were incubated with appropriate secondary HRP-conjugated secondary antibodies (Santa Cruz Biotechnology, Inc., Heidelberg, Germany) for $30 \mathrm{~min}$ at RT and finally washed as described above. Membranes were finally subjected to ECL reaction with a blotting substrate (Advansta, CA, USA, 12045-D20).

\subsection{Antibodies}

The following primary antibodies were used for Western blot analysis: rabbit polyclonal anti-PARP1(1:1000) (Proteintech, Rosemont, IL, USA, \#13371-1), rabbit polyclonal anti-phospho STAT3 Tyr705 (1:500) (Santa Cruz Biotechnology, Inc. Heidelberg, Germany, \#sc-8059), mouse monoclonal anti-STAT3 (1:100) (BD Transduction Lab, Franklin Lakes, NJ, USA, \#610189), rabbit polyclonal anti-LC3I/II (1:1000) (Novus, CO, USA, \#NB1002220), mouse monoclonal anti-p62/SQSTM1 (1:300) (BD Transduction Lab, \#610832), rabbit polyclonal anti-XBP1 (1:1000) (NovusBio, \#NBP1-77681SS), rabbit polyclonal anti-ATF6 (1:200) (Cell Signaling Technology, Danvers, MA, USA, \#65880), rabbit polyclonal antiphospho eIF2 $\alpha$ (Ser15) (1:200) (Cell Signaling, \#3398), rabbit polyclonal anti-eIF2 $\alpha$ (1:500) (Cell Signaling, \#9722), mouse monoclonal anti- $\beta$-actin (1:10000) (Sigma Aldrich, \#A5441) and anti hsp70 (Santa Cruz Biotechnology, Inc. Heidelberg, Germany, \#sc-32239) were used as loading control. Goat anti-rabbit IgG-horseradish peroxidase HRP (1:10000) (Santa Cruz Biotechnology, Inc. Heidelberg, Germany, sc-2004), goat anti-mouse IgG-horseradish peroxidase HRP (1:10000) (Santa Cruz Biotechnology, Inc. Heidelberg, Germany, sc-2005) were used as secondary antibodies. All primary and secondary antibodies used in this study were diluted in a PBS- $0.2 \%$ Tween 20 solution containing 3\% BSA.

\subsection{Densitometric Analysis}

Densitometric analysis of Western blot bands was performed by using the ImageJ software, which was downloaded from the NIH web site (http:/ / imagej.nih.gov, version 1.41o, NIH, USA).

\subsection{Sub-G1 Cell Cycle Analysis}

For cell cycle analysis, the DNA content was measured by Propidium Iodide (Sigma Aldrich, USA; P4170) staining and FACS analysis. PEL cells untreated (CT) and treated with GSK $(20 \mathrm{ng} / \mathrm{mL}), 4 \mu 8 \mathrm{C}(20 \mu \mathrm{M})$ and Ceapin $(12 \mu \mathrm{M})$ for $24 \mathrm{~h}$ were washed with cold $1 \times$ PBS and fixed in $70 \%$ ethanol on ice for at least $1 \mathrm{~h}$. After centrifugation, each cell pellet was washed with cold $1 \times$ PBS and stained with $50 \mu \mathrm{g} / \mathrm{mL}$ PI and RNase for 15 min at $37^{\circ} \mathrm{C}$. DNA content was measured by a BD Biosciences FACSCalibur. Data are representative of at least three independent experiments.

\subsection{Chemiluminescen Immunometric Assay}

After $24 \mathrm{~h}$ in which BC3 and BCBL1 were cultured with $4 \mu 8 \mathrm{C} 20 \mu \mathrm{M}$ or Ceapin $12 \mu \mathrm{M}$ or GSK2606414 $20 \mathrm{ng} / \mathrm{mL}$ or untreated (CT), cells were centrifuged, and supernatants were collected to measure Interleukin-10 (IL-10), Interleukin-6 (IL-6) and VEGF by Magnetic Luminex assay performed by R\&D systems a Bio-Techne brand, using a human premixed multi-analyte kit (R\&D systems Bio-Techne, LXSAHM) according to the manufacturer's instructions. 


\subsection{RNA Interference}

XBP1 silencing was performed by using specific small interfering RNA, according to the manufacturer's instructions (Santa Cruz Biotechnology, Inc., Heidelberg, Germany, sc38627). Briefly, $3 \times 10^{5}$ cells were seeded in six-well culture plates in RPMI medium without antibiotics for $24 \mathrm{~h}$. Next, $100 \mathrm{pmol}$ of specific small interfering RNA (siRNA duplex) or non-targeting (scramble) siRNA and $10 \mu \mathrm{L}$ of transfection reagent (Lipofectamine 2000; Invitrogen, CA, USA, 11668-027) were diluted in Opti-MEM medium (Thermo Fisher, Waltham, MA, USA, 31985062) and added to the cells according to the manufacturer's instruction [15]. After $24 \mathrm{~h}$, cells were collected, and the viability was assessed by trypan blue exclusion assay. Cells were harvested and used for Western blot analysis. Each experiment was performed in triplicate.

\subsection{Statistical Analysis}

Results are represented by the mean \pm standard deviation (SD) of at least three independent experiments, and a two-tailed Student's $t$-test was used to demonstrate statistical significance. A difference was considered as statistically significant when the $p$-value was at least $<0.05\left(^{*}\right)$.

\section{Results}

3.1. The Inhibition of IRE1alpha/XBP1 Axis by 4 48 C Strongly Reduces PEL Cell Survival While GSK2606414 PERK or Ceapin ATF6 Inhibitors Slightly Affect it

Previous studies have shown that PEL cells display a basal UPR activation [6], as they are secretory B cells characterized by a high level of ER stress. Therefore, we first inhibited IRE1alpha endoribonuclease activity by using $4 \mu 8 \mathrm{C}$. We found that it induced a dose-dependent cell death in both BC3 and BCBL1 PEL cells (Figure 1A). Differently from 4u8c, the inhibition of PERK by GSK2606414 or of ATF6 by Ceapin slightly reduced PEL cell survival (Figure 1A). We then evaluated whether these inhibitors at the doses used to treat PEL cells could effectively reduce the activation of the three UPR sensors. Therefore, we evaluated by Western blot analysis the spliced form of XBP1 (XBP1s), target of IRE1alpha endoribonuclease activity, the phosphorylation of eIF2alpha, target of PERK and the cleaved form ATF6, whose formation is inhibited by Ceapin, as it antagonizes ATF6 transport to the Golgi apparatus [16]. As shown in Figure 1B-D, the expression level of XBP1s and the cleaved form of ATF6 as well as the phosphorylation of eIF2alpha were reduced by using the UPR specific inhibitors, used at the highest dose. To explore whether the impairment of cell survival occurring following UPR inhibition, particularly following $4 \mu 8 \mathrm{C}$ treatment, could be due to apoptosis induction, we evaluated the cleavage of PARP1, as it can be mediated by caspase activation [17]. As shown in Figure 1E, the PARP1 cleaved form increased in PEL cells treated by $4 \mu 8 \mathrm{C}$, while it slightly increased following treatment with GSK2606414 or Ceapin. Apoptosis induction was confirmed by the increase in subG1 events that was particularly evident in $4 \mu 8 \mathrm{C}$-treated PEL cells (Figure 1F).

\subsection{4 $48 \mathrm{C}$ Reduces the Release of Pro-Inflammatory/Immune Suppressive Cytokines and STAT3 Activation in PEL Cells}

Searching for the molecular mechanism/s that could lead to higher cell death induction by $4 \mu 8 \mathrm{C}$, we assessed the release of cytokines IL- 6 , IL-10 and VEGF, known to sustain PEL cell survival following treatment with $4 \mu 8 \mathrm{C}$ or GSK2606414 or Ceapin. The results shown in Figure $2 \mathrm{~A}$ indicate that only $4 \mu 8 \mathrm{C}$ strongly reduced the production of these cytokines, which was slightly downregulated by GSK2606414 or Ceapin. These cytokines may lead to the activation of STAT3, a pathway whose constitutive phosphorylation plays a pro-survival role in PEL. We found that STAT3 705 tyrosine phosphorylation (Tyr705) was reduced by $4 \mu 8 \mathrm{C}$ (Figure $2 \mathrm{~B}$ ), according to its mediated impairment of cytokine release. All together, these results suggest that the reduction in cytokine production and STAT3 de-phosphorylation could underlie the impairment of PEL cell survival mediated by $4 \mu 8 \mathrm{C}$. 
BC3

A

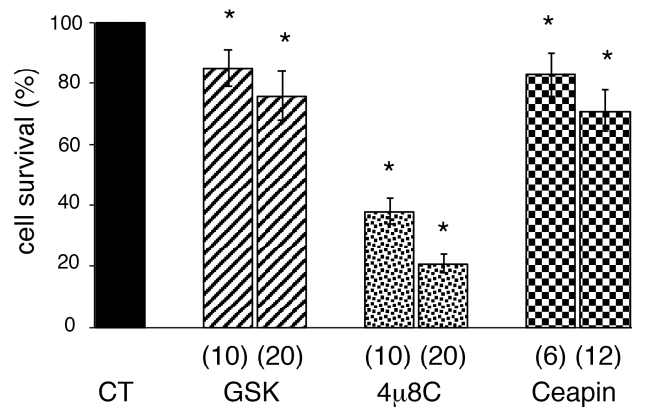

BCBL1

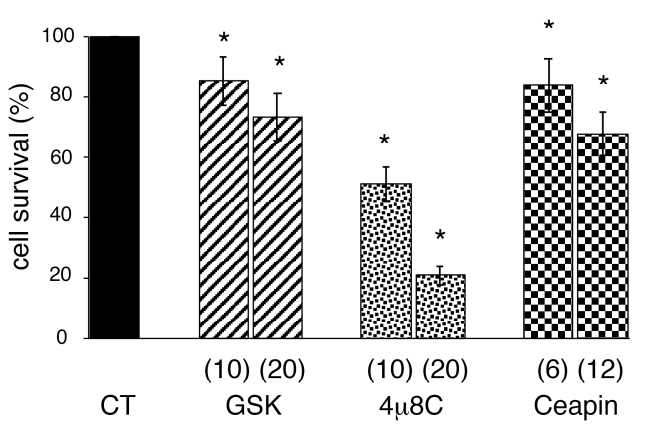

B

E

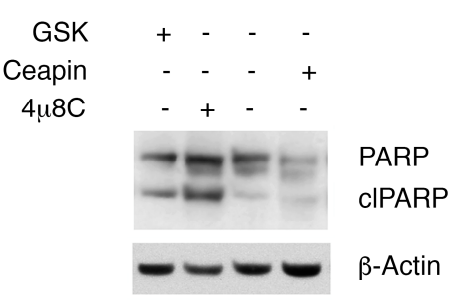

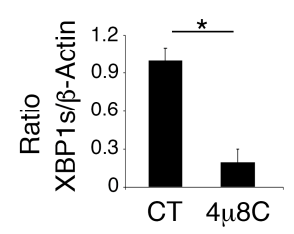

C
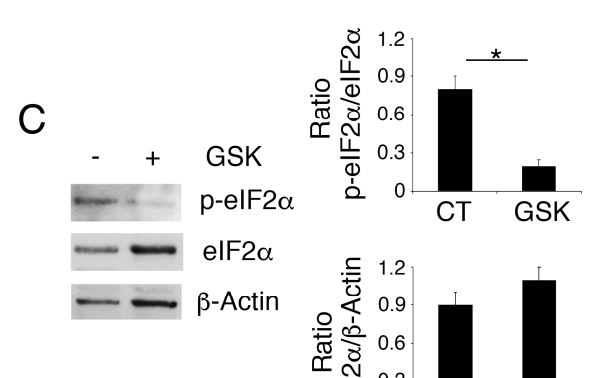

D

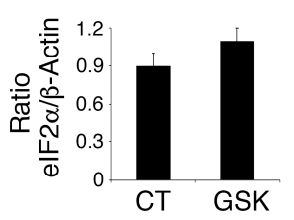

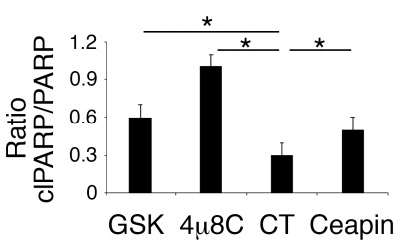

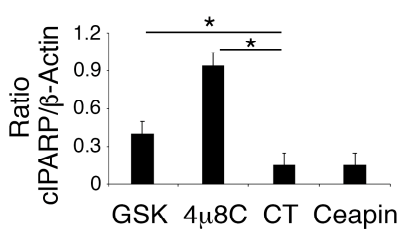

$\mathrm{F}$

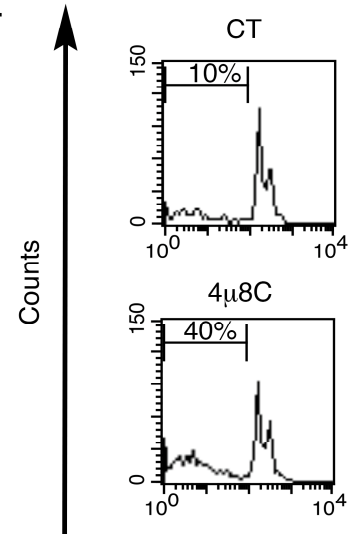

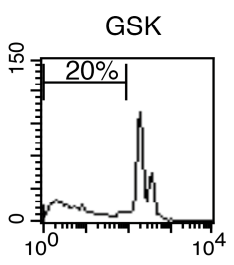

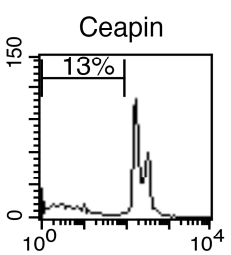

DNA content

Figure 1. $4 \mu 8 \mathrm{C}$ strongly impairs PEL cell survival while GSK2606414 or Ceapin slightly reduce it. BC3 and BCBL1 were

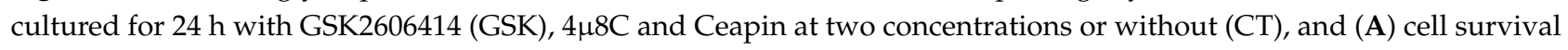
was evaluated by trypan blue exclusion assay. The histograms represent the percentage of cell viability relative to the control; data are represented as the mean plus SD of more than 3 experiments ${ }^{*} p$ value $<0.05$. The expression of XBP1 (B), p-eIF2alpha and eIF2alpha (C) and ATF6 (D) inhibited by their respective inhibitor $4 \mu 8 \mathrm{C}(20 \mu \mathrm{M}), \mathrm{GSK}(20 \mathrm{ng} / \mathrm{mL})$ and Ceapin $(12 \mu \mathrm{M})$ was evaluated by Western blot analysis in BCBL1 PEL cells. Actin was used as loading control. A representative experiment out of three is shown. Histograms represent the mean plus SD of the densitometric analysis of the ratio of specific protein/ $\beta$-actin. (E) Cleavage of PARP was evaluated by Western blot analysis after treatment of PEL cells with GSK $(20 \mathrm{ng} / \mathrm{mL}), 4 \mu 8 \mathrm{C}(20 \mu \mathrm{M})$ and Ceapin $(12 \mu \mathrm{M})$ or without $(\mathrm{CT})$. Actin was used as loading control. A representative experiment out of three is shown. Histograms represent the mean plus SD of the densitometric analysis of the ratio of clPARP/PARP and clPARP/ $\beta$-actin. ${ }^{*} p$-value $<0.05$. (F) sub-G1of untreated (CT) and treated with GSK $(20 \mathrm{ng} / \mathrm{mL}), 4 \mu 8 \mathrm{C}(20 \mu \mathrm{M})$ and Ceapin $(12 \mu \mathrm{M})$ PEL cells was evaluated by FACS analysis after staining with PI. \% of sub G1 is reported inside the histograms. 
A
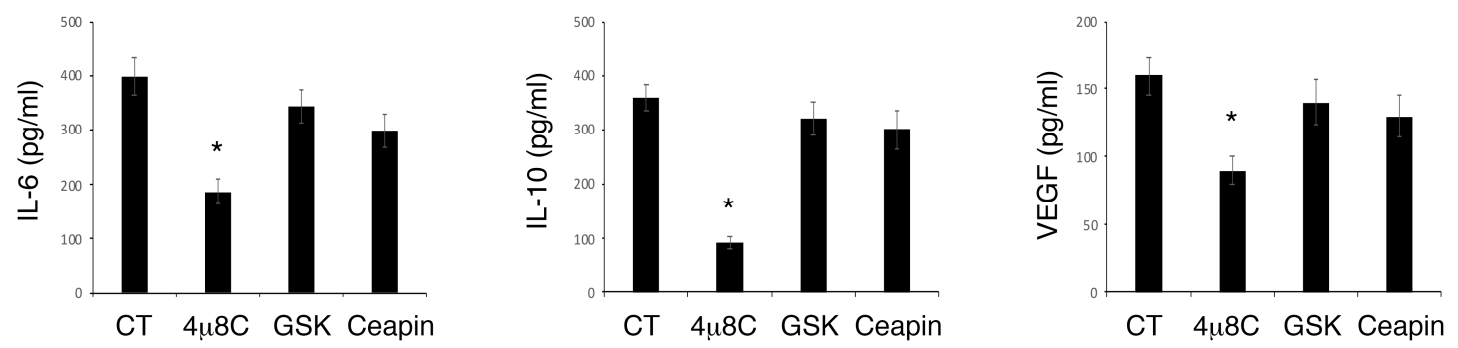

B

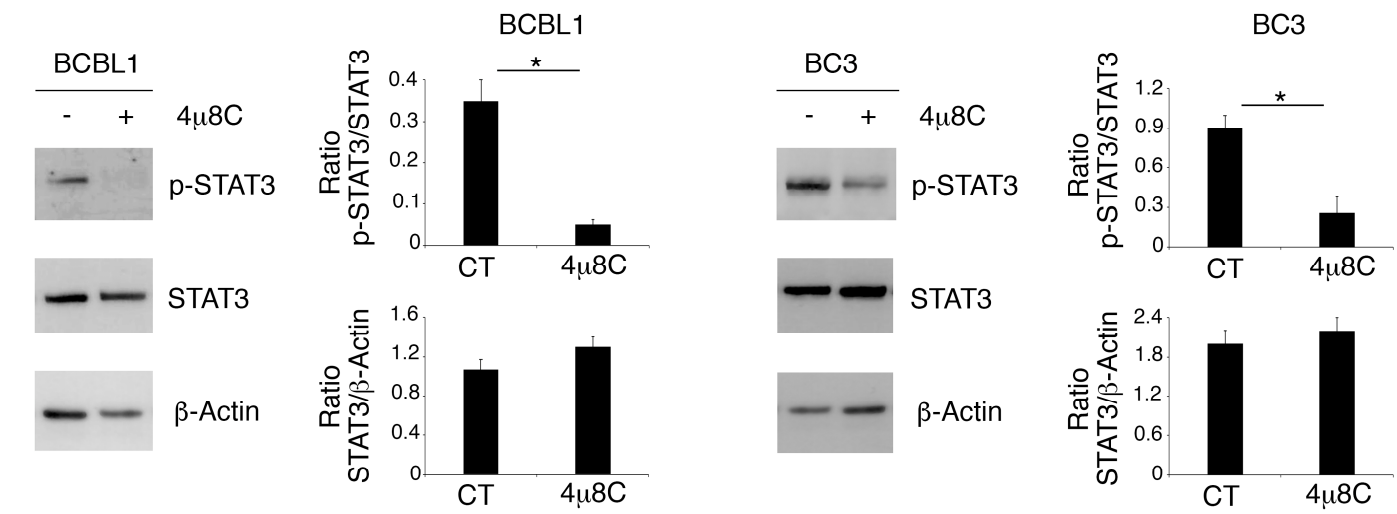

Figure 2. $4 \mu 8 \mathrm{C}$ reduces pro-inflammatory/immune suppressive cytokines and inhibits STAT3 phosphorylation in PEL cells. Release of IL-6, IL-10 and VEGF was measured in supernatants of PEL cells treated with $4 \mu 8 \mathrm{C}(20 \mu \mathrm{M}), \mathrm{GSK}(20 \mathrm{ng} / \mathrm{mL})$ and Ceapin $(12 \mu \mathrm{M})$ or without (CT), (A). Histograms representing the mean plus SD of the amount of IL-6, IL-10 and VEGF of three independent experiments are shown. * $p$-value $<0.05$. (B) The expression of p-STAT3 and STAT3 in BCBL1 and BC3 cells treated or not $(\mathrm{CT})$ with $4 \mu 8 \mathrm{C}(20 \mu \mathrm{M})$ was evaluated by Western blot analysis. Actin was used as loading control. A representative experiment out of three is shown. Histograms represent the mean plus SD of the densitometric analysis of the ratio of $\mathrm{p}$-STAT3/STAT3 and STAT3/ $\beta$-actin. * $p$-value $<0.05$.

\subsection{4 $48 \mathrm{C}$ Induces Autophagy Whose Inhibition Increases Its Cytotoxicity against PEL Cells}

We then evaluated the impact of UPR sensor inhibitors on autophagy. As shown in Figure 3A,B, while $4 \mu 8 \mathrm{C}$ reduced the expression level of p62/SQSTM1, GSK2606414 and Ceapin promoted its accumulation. As p62/SQSTM1 is mainly degraded through autophagy, these results suggest that $4 \mu 8 \mathrm{C}$ promoted the autophagic flux, while the other two UPR inhibitors reduced it. The induction of autophagy by $4 \mu 8 \mathrm{C}$ was confirmed by its combination with Cloroquine, a drug that inhibits the late autophagic steps. We found that the expression level of the lipidated form of LC3 (LC3II) increased with $4 \mu 8 \mathrm{C}$ and further accumulated by using Cloroquine (Figure 3C). As LC3II is formed and degraded through autophagy, preventing its degradation by Cloroquine allows us to evaluate LC3II formation [18]. Therefore, the higher LC3II increase in the presence of $4 \mu 8 \mathrm{C}$ plus Cloroquine, together with the reduction in p62 expression level, suggests that $4 \mu 8 \mathrm{C}$ induced a complete autophagic flux in PEL cells. We took advantage of using Cloroquine to also evaluate the role of autophagy activation by $4 \mu 8 \mathrm{C}$ on cell survival. As shown in Figure 3D, PEL cell survival impaired by $4 \mu 8 \mathrm{C}$ was further reduced by its combination with Cloroquine, suggesting that autophagy activation by $4 \mu 8 \mathrm{C}$ represented a pro-survival mechanism. 
A
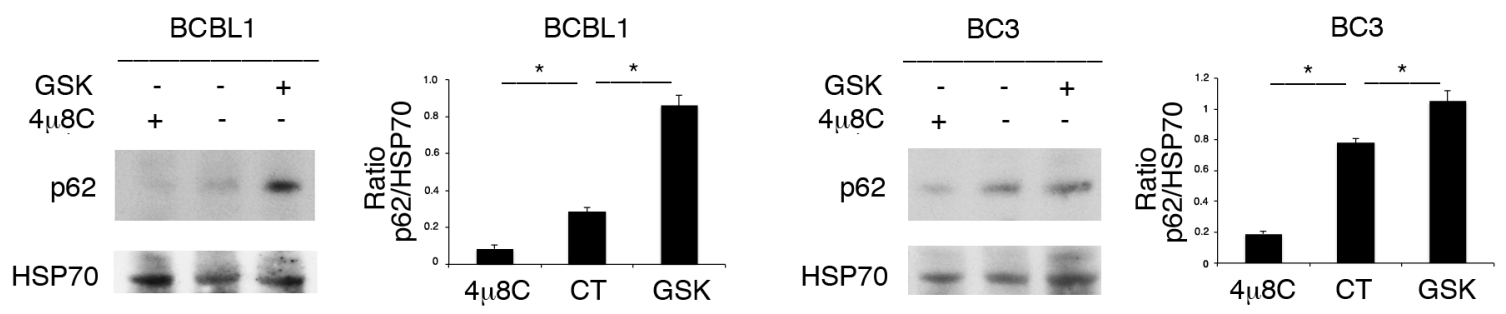

B
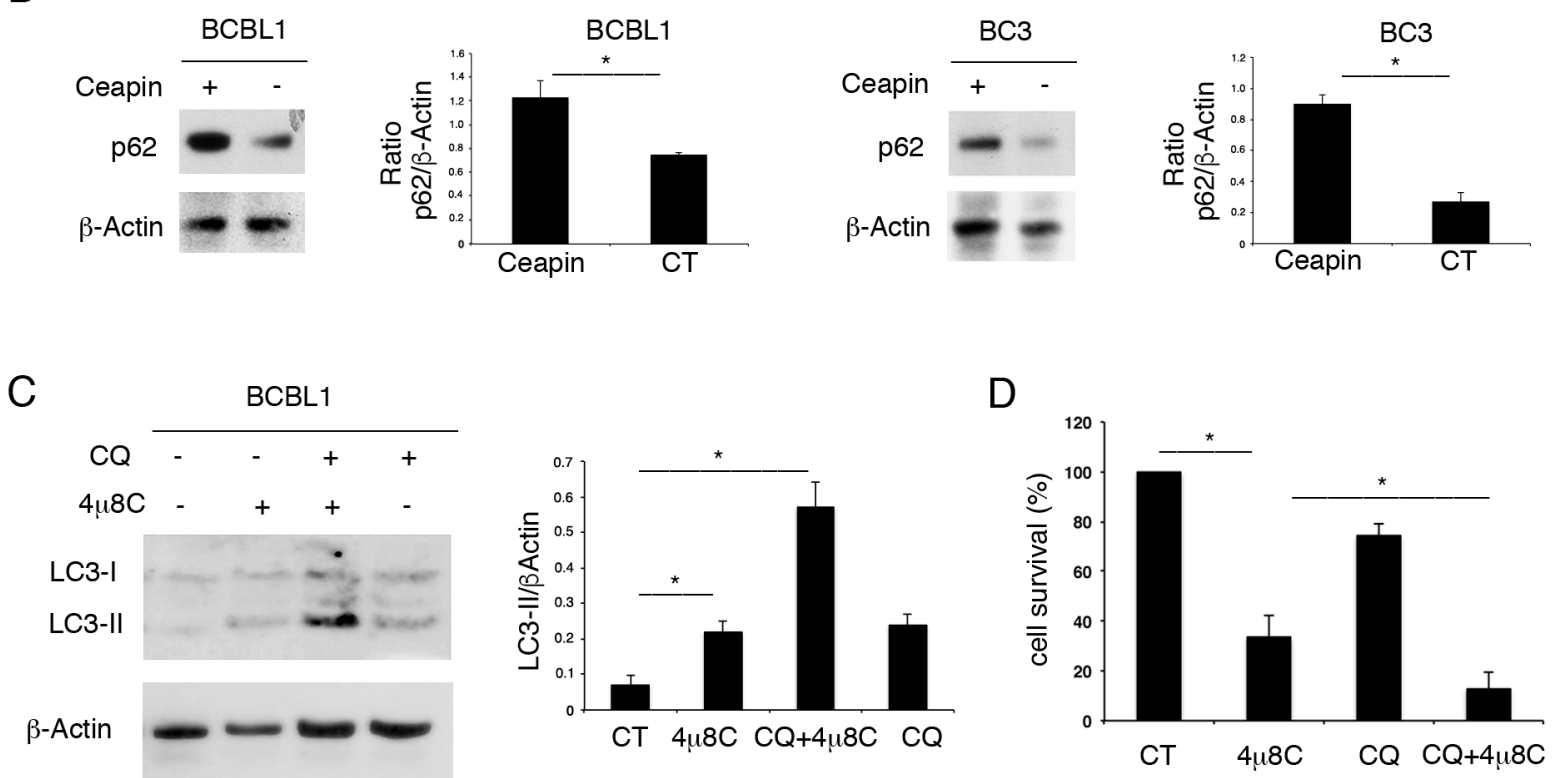

Figure 3. $4 \mu 8 \mathrm{C}$ promotes a pro-survival autophagy in PEL cells. (A) BC3 and BCBL1 cells were treated with $4 \mu 8 \mathrm{C}$ or with GSK2606414 (GSK) or (B) with Ceapin and p62/SQSTM1 (p62) expression level was evaluated by Western blot. (C) LC3I/II was evaluated by Western blot analysis in BCBL1 PEL cells treated by $4 \mu 8 \mathrm{C}$ in the presence or in the absence of the autophagy inhibitor Cloroquine (CQ). A representative experiment is shown. Histograms represent the mean plus SD of the densitometric analysis of three independent experiments. ${ }^{*} p$-value $<0.05$. (D) Cell survival was evaluated by trypan blue exclusion assay in PEL cells treated by $4 \mu 8 \mathrm{C}$ in the presence or in the absence of the autophagy inhibitor Cloroquine. Histograms represent the mean plus SD of three independent experiments. ${ }^{*} p$-value $<0.05$.

\subsection{XBP1 Silencing Induces Autophagy and Reduces Cells Survival in PEL Cells}

The role of XBP1 inhibition in autophagy and survival of PEL cells was then evaluated by silencing it by specific siRNA. We found that XBP1 knock-down (Figure 4A) reduced p62/SQSTM1 expression level in comparison with scramble-treatment, suggesting autophagy activation by XBP1 silencing (Figure 4B). Moreover, similarly to what was observed by using $4 \mu 8 \mathrm{C}$, XBP1 silencing increased LC3II expression level (Figure 4B) and reduced PEL cell survival (Figure 4C). 

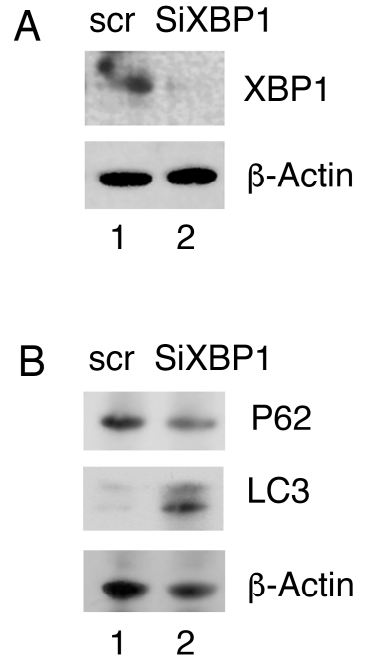
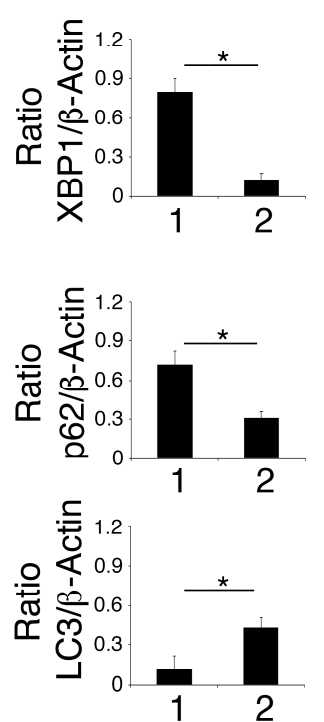

C

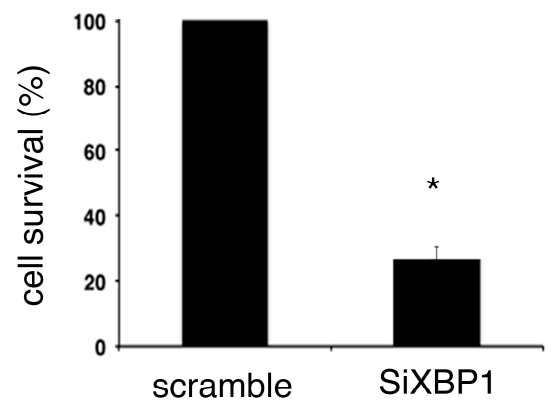

Figure 4. XBP1 knock-down promotes autophagy and impairs cell survival in PEL cells. The expression of XBP1, p62 and LC3 was evaluated by Western blot analysis in PEL cells silenced with siRNA XBP1 and control cells (scramble, scr) (A,B). Actin was used as loading control. A representative experiment out of three is shown. Histograms represent the mean plus SD of the densitometric analysis of the ratio of XBP1/ $\beta$-actin, p62/ $\beta$-actin and LC3/ $\beta$-actin. * $p$ value $<0.05$. (C) cell survival of PEL cells silenced with siRNA XBP1 and control cells (scramble) was evaluated by trypan blue exclusion assay. The histograms represent the percentage of cell viability relative to the control; data are represented as the mean plus SD of more than 3 experiments * $p$ value $<0.05$.

\section{Discussion}

Tumor cells, especially those that are highly secretory, such as the cancer B cells belonging to Multiple Myeloma (MM) or PEL, are characterized by a high level of basal ER stress and a constitutive UPR activation. As such, these cells are very sensitive to ER perturbation; therefore, they may be successfully treated with drugs that exacerbate ER stress, such as HSPs inhibitors [19] that reduce the folding capacity of the cells or Bortezomib [7], which, by inhibiting the proteasome, further increases the already high protein load in the ER. However, UPR may represent a rational drug target for cancer that strongly relies on its activation, and indeed UPR inhibition may be a promising anticancer strategy $[20,21]$, considering that UPR triggers pro-survival processes such as the upregulation of chaperones or autophagy. However, drugs that exacerbate ER stress/UPR can also have a cytotoxic effect on cancer cells [22], suggesting that the activation of UPR in stressed cancer cells must be well balanced as it may drive cells towards either death or survival, depending on the duration, intensity and nature of stress. Of note, the response initiated by the three UPR sensors is interconnected either in a positive way, as when they converge on the activation of the same targets, or in a negative way, as the activation of a sensor may inhibit the other/s [23,24].

In this study, we found that PEL cells were more highly dependent on the function of the IRE1alpha/XBP1 axis rather than PERK and ATF6 branches. Indeed, IRE1alpha/XBP1 axis inhibition by $4 \mu 8 \mathrm{C}$ decreased the production of cytokines such as IL-6, IL-10 and VEGF, while such process was slightly influenced by GSK2606414 or Ceapin, PERK and ATF6 inhibitors. The dependence of PEL from the release of these cytokines for its survival has been clearly shown in previous studies [25]. Indeed, it must be considered that these cytokines may activate, in an autocrine fashion, transcription factors such as STAT3 in PEL cell [26] and that its constitutive activation plays a key role in sustaining PEL survival [4]. Accordingly, here, we found that in addition to impairing cytokine production, Ire1alpha/XBP1 axis inhibition led to a reduction in STAT3 phosphorylation in these cells. The close relationship between IRE1alpha/XBP1 axis activation and pro- 
inflammatory/immune suppressive cytokine secretion has been recently shown by our laboratory in macrophages infected by KSHV [27], the same virus carried by PEL cells and involved in the etiology of this lymphoma. Moreover, IL-6, IL-10 and VEGF, produced by cancer cells, may activate STAT3 in a paracrine fashion in the cells present in the tumor microenvironment, such as myeloid cells or fibroblasts, transforming them into cells that support tumor growth [28]. Therefore, it will be important, in future studies, to evaluate the consequence of IRE1alpha/XBP1 axis inhibition on the cross-talk between PEL cells and cells of the tumor environment or whether IRE-XBP1 signaling inhibition may interfere with HIF1 $\alpha$. Indeed, it is known that the interaction between these pathways may sustain cancer growth in hypoxic condition or promote the epithelial to mesenchymal transition (EMT) [29]. However, the importance of targeting the IRE1alpha/XBP1 axis in vivo and in vitro has been reported by a variety of studies, particularly those focused on hematological cancers. In addition to $4 \mu 8 \mathrm{C}$, several small molecules inhibiting this axis, such as MKC-3946 and STF-083010, have been used [30,31]. Another important finding of this study was that IRE1alpha/XBP1 axis inhibition triggered autophagy in PEL cells and that its inhibition by Cloroquine could further promote PEL cell death. Inhibiting autophagy, particularly in the course of anticancer treatments, such as those that inhibit STAT3, has been previously shown to promote PEL cell death $[4,5]$. Indeed, it known that autophagy may help cells to adapt to stressful conditions including anticancer treatments. Based on this evidence, various drugs targeting both UPR and autophagy are currently in preclinical and clinical trials [32]. As autophagy also influences the release of Damage Associated Molecular Patterns (DAMPs) [33], it will be also important to evaluate how the IRE-XBP1 axis may shape the tumor environment by modulating autophagy [34,35].

In conclusion, this study suggests that targeting UPR, particularly the IRE1alpha/XBP1 axis, may represent a promising strategy against PEL and that its cytotoxic effect may be increased by combing it with autophagy inhibitors.

Author Contributions: R.G.: investigation and formal analysis, M.S.G.M.: investigation and data curation, L.G.: methodology, M.A.R.: software and validation, R.S.: validation and M.C.: funding acquisition, supervision, project administrator, writing-review and editing. All authors have read and agreed to the published version of the manuscript.

Funding: This work was supported by grants from Istituto Pasteur Italia-Fondazione Cenci Bolognetti, PRIN 2017 (2017K55HLC) and by the Italian Association for Cancer Research (AIRC) Grant (IG 2019 Id.23040).

Institutional Review Board Statement: The research has been performed in accordance with the Declaration of Helsinki and has been approved by the ethic committee of Policlinico Umberto I, Rome, Italy $(847 / 19)$.

Data Availability Statement: The data presented in this study are available on request from the corresponding author.

Conflicts of Interest: The authors declare no conflict of interest. The funders had no role in the design of the study; in the collection, analyses, or interpretation of data; in the writing of the manuscript, or in the decision to publish the results.

\footnotetext{
Abbreviations

Activating transcription factor (ATP); Endoplasmic Reticulum (ER); Epstein-Barr virus (EBV); Fasassociated with death domain (FADD)-like interleukin-1 $\beta$-converting enzyme (FLICE)/ caspase 8inhibitory protein (FLIP); interleukin (IL); inositol requiring kinase (IRE); Kaposi's Sarcoma-associated Herpesvirus (KSHV); Microtubule-associated protein 1A/1B-light chain 3 (LC3); multiple myeloma (MM); Nuclear factor-kB (NF-kB); nuclear factor erythroid 2-related factor (NRF2); p62, sequestosome 1 (SQSTM1); Poly (ADP-ribose) polymerase 1 (PARP1); Primary Effusion Lymphoma (PEL); double stranded RNA-activated protein kinase-like ER kinase (PERK); Signal Transducer and activator of Transcription 3 (STAT3); Unfolded Protein Response (UPR); Vascular-Endothelial Growth Factor
} 
(VEGF); X-box binding protein 1 (XBP1).

\section{References}

1. Thome, M.; Schneider, P.; Hofmann, K.; Fickenscher, H.; Meinl, E.; Neipel, F.; Mattmann, C.; Burns, K.; Bodmer, J.L.; Schroter, M.; et al. Viral FLICE-inhibitory proteins (FLIPs) prevent apoptosis induced by death receptors. Nature 1997, 386, 517-521. [CrossRef] [PubMed]

2. Cirone, M. EBV and KSHV infection dysregulates autophagy to optimize viral replication, prevent immune recognition and promote tumorigenesis. Viruses 2018, 10, 599. [CrossRef] [PubMed]

3. Klein, U.; Gloghini, A.; Gaidano, G.; Chadburn, A.; Cesarman, E.; Dalla-Favera, R.; Carbone, A. Gene expression profile analysis of AIDS-related primary effusion lymphoma (PEL) suggests a plasmablastic derivation and identifies PEL-specific transcripts. Blood 2003, 101, 4115-4121. [CrossRef] [PubMed]

4. Granato, M.; Chiozzi, B.; Filardi, M.R.; Lotti, L.V.; Di Renzo, L.; Faggioni, A.; Cirone, M. Tyrosine kinase inhibitor tyrphostin AG490 triggers both apoptosis and autophagy by reducing HSF1 and Mcl-1 in PEL cells. Cancer Lett. 2015, 366, $191-197$. [CrossRef]

5. Granato, M.; Rizzello, C.; Gilardini Montani, M.S.; Cuomo, L.; Vitillo, M.; Santarelli, R.; Gonnella, R.; D’Orazi, G.; Faggioni, A.; Cirone, M. Quercetin induces apoptosis and autophagy in primary effusion lymphoma cells by inhibiting PI3K/AKT/mTOR and STAT3 signaling pathways. J. Nutr. Biochem. 2017, 41, 124-136. [CrossRef]

6. Sarosiek, K.A.; Cavallin, L.E.; Bhatt, S.; Toomey, N.L.; Natkunam, Y.; Blasini, W.; Gentles, A.J.; Ramos, J.C.; Mesri, E.A.; Lossos, I.S. Efficacy of bortezomib in a direct xenograft model of primary effusion lymphoma. Proc. Natl. Acad. Sci. USA 2010, 107, 13069-13074. [CrossRef]

7. Granato, M.; Santarelli, R.; Lotti, L.V.; Di Renzo, L.; Gonnella, R.; Garufi, A.; Trivedi, P.; Frati, L.; D’Orazi, G.; Faggioni, A.; et al. JNK and macroautophagy activation by bortezomib has a pro-survival effect in primary effusion lymphoma cells. PLoS ONE 2013, 8, e75965. [CrossRef]

8. Hwang, J.; Qi, L. Quality control in the endoplasmic reticulum: Crosstalk between ERAD and UPR pathways. Trends Biochem. Sci. 2018, 43, 593-605. [CrossRef]

9. Papandreou, I.; Denko, N.C.; Olson, M.; Van Melckebeke, H.; Lust, S.; Tam, A.; Solow-Cordero, D.E.; Bouley, D.M.; Offner, F.; Niwa, M.; et al. Identification of an Ire1alpha endonuclease specific inhibitor with cytotoxic activity against human multiple myeloma. Blood 2011, 117, 1311-1314. [CrossRef]

10. Cirone, M. Could UPR manipulation help to tune the inflammatory response in the course of COVID-19? Virol. Immunol. J. 2020. [CrossRef]

11. D'Orazi, G.; Cordani, M.; Cirone, M. Oncogenic pathways activated by pro-inflammatory cytokines promote mutant p53 stability: Clue for novel anticancer therapies. Cell. Mol. Life Sci. 2020. [CrossRef] [PubMed]

12. Meares, G.P.; Liu, Y.; Rajbhandari, R.; Qin, H.; Nozell, S.E.; Mobley, J.A.; Corbett, J.A.; Benveniste, E.N. PERK-Dependent activation of JAK1 and STAT3 contributes to endoplasmic reticulum stress-induced inflammation. Mol. Cell. Biol. 2014, 34, 3911-3925. [CrossRef] [PubMed]

13. Cirone, M. Perturbation of bulk and selective macroautophagy, abnormal UPR activation and their interplay pave the way to immune dysfunction, cancerogenesis and neurodegeneration in ageing. Ageing Res. Rev. 2020, 58, 101026. [CrossRef]

14. Gilardini Montani, M.S.; Granato, M.; Cuomo, L.; Valia, S.; Di Renzo, L.; D’Orazi, G.; Faggioni, A.; Cirone, M. High glucose and hyperglycemic sera from type 2 diabetic patients impair DC differentiation by inducing ROS and activating Wnt/beta-catenin and p38 MAPK. Biochim. Biophys. Acta 2016, 1862, 805-813. [CrossRef] [PubMed]

15. Garufi, A.; Ricci, A.; Trisciuoglio, D.; Iorio, E.; Carpinelli, G.; Pistritto, G.; Cirone, M.; D'Orazi, G. Glucose restriction induces cell death in parental but not in homeodomain-interacting protein kinase 2-depleted RKO colon cancer cells: Molecular mechanisms and implications for tumor therapy. Cell Death Dis. 2013, 4, e639. [CrossRef]

16. Gallagher, C.M.; Walter, P. Ceapins inhibit ATF6alpha signaling by selectively preventing transport of ATF6alpha to the Golgi apparatus during ER stress. Elife 2016, 5. [CrossRef] [PubMed]

17. Margolin, N.; Raybuck, S.A.; Wilson, K.P.; Chen, W.; Fox, T.; Gu, Y.; Livingston, D.J. Substrate and inhibitor specificity of interleukin-1 beta-converting enzyme and related caspases. J. Biol. Chem. 1997, 272, 7223-7228. [CrossRef]

18. Granato, M.; Santarelli, R.; Filardi, M.; Gonnella, R.; Farina, A.; Torrisi, M.R.; Faggioni, A.; Cirone, M. The activation of KSHV lytic cycle blocks autophagy in PEL cells. Autophagy 2015, 11, 1978-1986. [CrossRef]

19. Granato, M.; Lacconi, V.; Peddis, M.; Lotti, L.V.; Di Renzo, L.; Gonnella, R.; Santarelli, R.; Trivedi, P.; Frati, L.; D’Orazi, G.; et al. HSP70 inhibition by 2-phenylethynesulfonamide induces lysosomal cathepsin D release and immunogenic cell death in primary effusion lymphoma. Cell Death Dis. 2013, 4, e730. [CrossRef]

20. Walczak, A.; Gradzik, K.; Kabzinski, J.; Przybylowska-Sygut, K.; Majsterek, I. The role of the ER-induced UPR pathway and the efficacy of its inhibitors and inducers in the inhibition of tumor progression. Oxid. Med. Cell. Longev. 2019, 2019, 5729710. [CrossRef]

21. White-Gilbertson, S.; Hua, Y.; Liu, B. The role of endoplasmic reticulum stress in maintaining and targeting multiple myeloma: A double-edged sword of adaptation and apoptosis. Front. Genet. 2013, 4, 109. [CrossRef] [PubMed] 
22. Cerezo, M.; Lehraiki, A.; Millet, A.; Rouaud, F.; Plaisant, M.; Jaune, E.; Botton, T.; Ronco, C.; Abbe, P.; Amdouni, H.; et al. Compounds triggering ER stress exert anti-melanoma effects and overcome BRAF inhibitor resistance. Cancer Cell 2016, 29, 805-819. [CrossRef] [PubMed]

23. Iwakoshi, N.N.; Lee, A.H.; Vallabhajosyula, P.; Otipoby, K.L.; Rajewsky, K.; Glimcher, L.H. Plasma cell differentiation and the unfolded protein response intersect at the transcription factor XBP-1. Nat. Immunol. 2003, 4, 321-329. [CrossRef] [PubMed]

24. Okada, T.; Yoshida, H.; Akazawa, R.; Negishi, M.; Mori, K. Distinct roles of activating transcription factor 6 (ATF6) and doublestranded RNA-activated protein kinase-like endoplasmic reticulum kinase (PERK) in transcription during the mammalian unfolded protein response. Biochem. J. 2002, 366, 585-594. [CrossRef] [PubMed]

25. Gasperini, P.; Tosato, G. Targeting the mammalian target of Rapamycin to inhibit VEGF and cytokines for the treatment of primary effusion lymphoma. Leukemia 2009, 23, 1867-1874. [CrossRef]

26. Niemand, C.; Nimmesgern, A.; Haan, S.; Fischer, P.; Schaper, F.; Rossaint, R.; Heinrich, P.C.; Muller-Newen, G. Activation of STAT3 by IL- 6 and IL-10 in primary human macrophages is differentially modulated by suppressor of cytokine signaling 3 . J. Immunol. 2003, 170, 3263-3272. [CrossRef]

27. Gilardini Montani, M.S.; Falcinelli, L.; Santarelli, R.; Granato, M.; Romeo, M.A.; Cecere, N.; Gonnella, R.; D’Orazi, G.; Faggioni, A.; Cirone, M. KSHV infection skews macrophage polarisation towards M2-like/TAM and activates Ire1 alpha-XBP1 axis up-regulating pro-tumorigenic cytokine release and PD-L1 expression. Br. J. Cancer 2020, 123, 298-306. [CrossRef]

28. Schaafsma, E.; Yuan, Y.; Zhao, Y.; Cheng, C. Computational STAT3 activity inference reveals its roles in the pancreatic tumor microenvironment. Sci. Rep. 2019, 9, 18257. [CrossRef]

29. Limonta, P.; Moretti, R.M.; Marzagalli, M.; Fontana, F.; Raimondi, M.; Montagnani Marelli, M. Role of endoplasmic reticulum stress in the anticancer activity of natural compounds. Int. J. Mol. Sci. 2019, 20, 961. [CrossRef]

30. Mimura, N.; Fulciniti, M.; Gorgun, G.; Tai, Y.T.; Cirstea, D.; Santo, L.; Hu, Y.; Fabre, C.; Minami, J.; Ohguchi, H.; et al. Blockade of XBP1 splicing by inhibition of IRE1alpha is a promising therapeutic option in multiple myeloma. Blood 2012, 119, 5772-5781. [CrossRef]

31. Tang, C.H.; Ranatunga, S.; Kriss, C.L.; Cubitt, C.L.; Tao, J.; Pinilla-Ibarz, J.A.; Del Valle, J.R.; Hu, C.C. Inhibition of ER stressassociated IRE-1/XBP-1 pathway reduces leukemic cell survival. J. Clin. Investig. 2014, 124, 2585-2598. [CrossRef] [PubMed]

32. Ojha, R.; Amaravadi, R.K. Targeting the unfolded protein response in cancer. Pharmacol. Res. 2017, 120, 258-266. [CrossRef]

33. Wang, Y.; Li, Y.B.; Yin, J.J.; Wang, Y.; Zhu, L.B.; Xie, G.Y.; Pan, S.H. Autophagy regulates inflammation following oxidative injury in diabetes. Autophagy 2013, 9, 272-277. [CrossRef] [PubMed]

34. Cirone, M.; Gilardini Montani, M.S.; Granato, M.; Garufi, A.; Faggioni, A.; D’Orazi, G. Autophagy manipulation as a strategy for efficient anticancer therapies: Possible consequences. J. Exp. Clin. Cancer Res. 2019, 38, 262. [CrossRef]

35. Masuelli, L.; Granato, M.; Benvenuto, M.; Mattera, R.; Bernardini, R.; Mattei, M.; d'Amati, G.; D'Orazi, G.; Faggioni, A.; Bei, R.; et al. Chloroquine supplementation increases the cytotoxic effect of curcumin against Her2/neu overexpressing breast cancer cells in vitro and in vivo in nude mice while counteracts it in immune competent mice. Oncoimmunology 2017, 6, e1356151. [CrossRef] 